\title{
A study of thin film solid phase microextraction methods for analysis of fluorinated benzoic acids in seawater
}

\author{
Ezel Boyac1 ${ }^{\mathrm{a}, 1}$, Krzysztof Gorynski ${ }^{\mathrm{a}, \mathrm{b}, 1}$ C. Ricardo Viteri ${ }^{\mathrm{c}, 1}$, Janusz Pawliszyn $^{\mathrm{a}^{*}}$
}

\author{
${ }^{a}$ Department of Chemistry, University of Waterloo, 200 University Avenue West, \\ Waterloo, Ontario, N2L 3G1, Canada \\ ${ }^{b}$ Department of Pharmacodynamics and Molecular Pharmacology, Faculty of Pharmacy, \\ Collegium Medicum in Bydgoszcz, Nicolaus Copernicus University in Toruń, Toruń, \\ Poland \\ ${ }^{\mathrm{c}}$ Entanglement Technologies, Inc. 42 Adrian Ct. Burlingame, CA 94010
}

* Corresponding author. Tel.: +1 519888 4641/+1 519885 1210; fax: +1 519746 0435/+15197460434.

E-mail address: janusz@uwaterloo.ca (J. Pawliszyn).

${ }^{1}$ These authors contributed equally to the work. 


\begin{abstract}
Fluorinated benzoic acids (FBAs) are frequently used as tracers by the oil industry to characterize petroleum reservoirs. The demand for fast, reliable, robust, and sensitive approaches to separate and quantify FBAs in produced water, both in laboratory and field conditions, has not been yet fully satisfied. In this study, for the first time, thin film solid phase microextraction (TF-SPME) is proposed as a versatile sample preparation tool for the determination of FBAs in produced water by pursing two different approaches. First, an automated high throughput TF-SPME method using solvent desorption for fast and simultaneouspreparation of multiple samples prior to liquid chromatographic separation and high resolution mass spectrometricdetection (LC-MS) of FBAs was demonstrated for routine laboratory analysis. This method was optimized in terms of extraction phase chemistry, sample $\mathrm{pH}$ and ionic strength, extraction/desorption times using two representative FBAs (4-FBA and 2,3,4,5-tetraFBA). It incorporates a relatively simple sample pretreatment involving $\mathrm{pH}$ adjustment prior to the TF-SPME, and obtained limits of quantification (LOQ) are at the $1.0 \mathrm{ng} \mathrm{mL}^{-1}$ level. Second, the applicability ofTFSPME for fast mass spectrometric (MS) determination of FBAs with omission of derivatization and gas chromatographic (GC) separation was proven. This second method consists of manual extractions of analytes from seawater samples with a thermally stable TF-SPME membrane and direct thermal desorption of the extracted FBAs to a MS via a thermal desorption unit (TDU). It was demonstrated that the TF-SPME extracts and thermally releases analytes quantitatively and with good reproducibility. This approach opens up the possibility for on-site measurements with portable analyzers.
\end{abstract}

\title{
Keywords
}

Fluorinated Benzoic Acids (FBAs), LC-MS Analysis, Thin Film Solid Phase Microextraction (TF-SPME), High Throughput Analysis, Sample Preparation 


\section{Introduction}

Secondary recovery is a process in which reservoir fluid is mobilized from an injection well toward a production well. Problems arising from fluid channeling through minifractures, faults, and high permeability streaks result in poor contact between reservoir and injection fluids, and consequently, in low hydrocarbon recovery [1]. Tracer testing has been proven to help overcome these challenges during the design, testing, and execution of fluid injection programs [2-4]. Tracers analyses of the distribution of residence times between wells, together with numerical simulations, provide detailed information on reservoir heterogeneity and connectivity, remaining oil saturation and its distribution, and estimates of how much of the reservoir fluid volume contacts the injection fluid $[2,4]$. Owing to their unique characteristics, fluorinated benzoic acids (FBAs) are the most widely used tracers in fluid injection campaigns; they are highly stable under reservoir conditions, fairly inert (no binding to the matrix), do not naturally occur in nature, and are found in several distinct structural compositions, which allows for their application towards multiple tracing experiments in fractured media where several conservative tracers are required [5-7]. For these reasons, FBAs are also utilized to trace groundwater movement in soil, and as indicators in leaching studies for carbon sequestration techniques [7].As they are typically injected in low quantities, several analytical methods have been proposed to enable the detection of these compounds at trace and ultra-trace levels [5,6].The lowest detection limits reported to date are below $0.050 \mathrm{ng} \mathrm{mL}^{-1}$ and are achieved by solid phase extraction (SPE) followed by gas chromatography mass spectrometric (GC-MS) analysis [8,9]. Although the obtained detection limits in this method are very good, it nonetheless requires many tedious sample preparation steps. For instance, in SPE, extraction of samples is followed by a long derivatization step $(24 \mathrm{~h})$ to convert FBAs into fluorobenzoic acid methyl esters (FBAMEs), and a solvent exchange step is further incorporated prior to the GC-MS analysis. As FBAs are nonvolatile and relatively polar, high pressure liquid chromatography (HPLC) offers the advantage of elimination of the derivatization step, speeding up direct analysis. However, to date, the reported HPLC-UV detection limits are typically two to three orders of magnitude higher than the current reference method; for instance, a HPLC-UV method with minor sample preparation can only offer detection 
limits as low as $10 \mathrm{ng} \mathrm{mL} \mathrm{m}^{-1}$ [3]. On the other hand, when HPLC is coupled toa more sensitive mass detector, superior detection capabilities can be achieved. For instance, high pressure liquid chromatography tandem mass spectrometry (LC-MS/MS) methods with limits of detection in the $\mathrm{ng} \mathrm{mL}^{-1}$ [7] and $0.05-50 \mathrm{ng} \mathrm{mL}^{-1}$ range [6] have been reported for FBAs. Recently, a study employing LC-MS based determination after SPE reported detection in the $0.016-0.210 \mathrm{ng} \mathrm{mL}^{-1}$ range, with an enrichment factor of 500, almost matching the sensitivity of the above-mentioned SPE followed by GC-MS method[8], while avoiding the tedious derivatization step [5]. Yet, despite the high enrichment factors achieved in these experiments, and the simultaneous clean-up of analytes from the matrix components offered by the method,its sample preparation methodology is as comparatively tedious as traditional SPE followed by GC-MS, and can be considered the bottleneck of the analysis. In addition, none of the reported methods scrutinize possible matrix effects that may occur in real sample analyses owing to salinity or other dissolved organics co-eluting with the probed analytes.

A novel approach to sample preparation is needed to simplify long procedures while still providing analyte selectivity in complex matrices. The bottleneck issue, often cited as a major challenge in chemical analyses, can be overcome by introducing a high throughput approach to sample preparation. In this sense, solid phase microextraction (SPME), which has of late been widely established in various areas as a method of choice, provides the opportunity for high throughput sampling and sample preparation. In SPME, the principle of extraction lies in the equilibrium established between the concentration of the analyte in the sample and the extraction phase [10]. SPME-based sampling and sample preparation approaches are well suited for automation [11,12], as well as direct coupling, or coupling via various interfaces to analytical instruments [13-15]. Additionally, SPME is flexible in applicable geometries and extraction chemistries, as well as suitable for in vivo and on site analysis. Among the various geometries that exist for SPME, thin film SPME (TF-SPME) has been recently introduced to further increase the sensitivity of the method. The basic principles of TF-SPME rely on the use of a relatively large surface volume for the extraction phase, while keeping the thickness of the coating similar to the traditional fiber coating thickness; this change in geometry practically improves the extracted amount of analytes in reasonable extraction times, 
which is ensured by fast sampling rates via contact of large extraction surface areas with the sample. Moreover, TF-SPME has been designed to fit commercially available 96-well plates, allowing for simultaneous sample preparation throughput of up to 96 samples. Considering its multiple benefits, TF-SPME has been proposed as a practical approach for high throughput sample preparation in bioanalysis [16-18], environmental analysis [19-21], clinical analysis [22-24] and doping analysis [25, 26], where many samples may be generated in a short time. The wide variety of TF-SPME applications available today stems from the simplicity of the SPME device; its re-usability, which decreases the analysis cost per sample; and its direct applicability to complex matrices without the necessity for sample pretreatment steps, such as filtration or centrifugation.

In this work, we present a method for analysis of FBAs tracers in brine based on TFSPME and liquid chromatography-high resolution mass spectrometry (LC-HRMS), with seawater introduced as a proxy for produced water. Limits of detection are below $1 \mathrm{ng}$ $\mathrm{mL}^{-1}$ for the two FBAs used during method development: 4-fluorinated benzoic acid (4FBA), and 2,3,4,5-tetrafluorinated benzoic acid (2,3,4,5-tetraFBA). The method requires minimal sample handling, and provides simultaneous sample preparation of up to96 samples, presenting obvious advantages over GC-MS techniques for aqueous samples that require derivatization. Additionally, the direct thermal desorption of analytes from TF-SPME to a quadrupole mass detector, which omits the necessity of derivatization, was scrutinized in order to emphasize the potential of TF-SPME for on-site sampling and analysis when portable detectors for this application become available. Direct SPME sampling in the field will aid in conserving sample integrity, and avoiding human error during handling and shipping of samples to analytical chemistry laboratories from remote oilfields. In addition, on-site sampling enables the representation of distributions of residence times of tracers between wells with higher point densities. 


\section{Experimental}

\subsection{Materials and chemicals}

All chromatographic solvents, namely water $\left(\mathrm{H}_{2} \mathrm{O}\right)$, acetonitrile $(\mathrm{ACN})$, and methanol $(\mathrm{MeOH})$, were of LC-MS grade, and purchased from Fisher Scientific (Ottawa, ON, Canada), while formic acid (FA) for mass spectrometry was obtained from Fluka (Oakville, ON, Canada). The analytes 4-FBA and 2,3,4,5-tetraFBAwere purchased from Aldrich (Oakville, ON, Canada). Individual standard stock solutions of $1 \mathrm{mg} \mathrm{mL}^{-1}$ of each FBA were prepared in $\mathrm{MeOH}$ and stored at $4^{\circ} \mathrm{C}$. A phosphate-buffered saline (PBS) solution ( $\mathrm{pH} 7.4$ ) was prepared in ultrapure water, as described elsewhere [12], by mixing appropriate amounts of sodium chloride, potassium chloride, potassium phosphate monobasic, and sodium phosphate dibasic (Sigma-Aldrich, Oakville, ON, Canada), and used as sample matrix in preliminary evaluations of optimum extraction phase. Synthetic seawater with a representative composition for the Gulf of Mexico (GOM) was prepared (following the recipe shown in Table 1S) by mixing appropriate amounts of sodium sulphate, potassium chloride, magnesium chloride hexahydrate, calcium chloride dehydrate (all from Sigma-Aldrich, Oakville, ON, Canada), and used in various stages of method development, as well as for validation of the final method. Working standard solutions were prepared daily by diluting the abovementioned analyte stock solutions in PBS or synthetic seawater.

In order to select the most suitable extraction phase for the study, various extraction phases and SPE particles were tested. Among the extraction phases tested, hydrophiliclipophilic balanced (HLB), C18, and strong anion exchange (SAX) particles were kindly obtained from Supelco (Bellefonte, PA, USA), while polystyrene divinylbenzene, modified with weak anion exchange (WAX-PS-DVB) particles, were purchased from Macherey-Nagel (Germany).To immobilize the particles on the surface of the blades, polyacrylonitrile (PAN) dissolved in N,N-dimethyloformamide (DMF) (both purchased from Sigma-Aldrich, Oakville, ON, Canada) was used as a glue by following the spraycoating method described by Mirnaghi et al. [27].

In addition to solvent desorption of extracted analytes from thin films, direct thermal desorption was also demonstrated. In this set-up, a thermal desorption unit (TDU) was directly coupled to a single quadrupole mass spectrometer. For these experiments, 
thermally stable membranes consisting of divinylbenzene (DVB), $(5 \mu \mathrm{m}$ diameter, Supelco, Bellefonte, PA, USA) immobilized in PDMS were prepared with the method described by Jiang et al. [28].

\subsection{Instrumentation}

\subsection{LC-MS instrumentation}

LC-MS analyses were performed in an Accela liquid chromatograph (equipped with a binary pump, a vacuum degasser, and a thermostated autosampler) coupled to an Exactive $^{\mathrm{TM}}$ benchtop Orbitrap mass analyzer with an electrospray ionization probe (ESI) (Thermo Scientific, San Jose, CA, USA).

Chromatographic separation of the analytes was achieved on a Discovery HS F5 column $\left(50 \mathrm{~mm} \times 2.1 \mathrm{~mm}\right.$ i.d., $3 \mu \mathrm{m}$; Supelco, Bellefonte, PA, USA) usinga $0.4 \mathrm{~mL} \mathrm{~min}^{-1}$ flow rate in a gradient elution, with water (solvent $\mathrm{A}$ ) and $\mathrm{ACN}$ (solvent $\mathrm{B}$ ) both acidified to contain $0.1 \%(\mathrm{v} / \mathrm{v})$ FA. The used gradient was as follows: for the first $0.5 \mathrm{~min}, 10 \% \mathrm{~B}$ was used, then raised to $70 \% \mathrm{~B}$ in $2.5 \mathrm{~min}$ and held for $0.5 \mathrm{~min}$, then returned to the initial settings in $0.5 \mathrm{~min}$, and held for $1 \mathrm{~min}$ at these conditions to ensure re-equilibration of the column. The injection volume was $10 \mu \mathrm{L}$ (full loop mode) for all analyses. The tray temperature of the autosampler was maintained at $5^{\circ} \mathrm{C}$. For evaluation of instrument

performance, quality control samples (QC) consisting of $50 \mathrm{ng} \mathrm{mL}^{-1}$ of each compound were injected periodically throughout the sequences, including in the beginning and end of each run.

Mass spectrometric analyses were performed in negative ionization mode with operating parameters set as reported on Table 2Sin Supplementary Information (SI). The mass resolution was set at 100,000 FWHM for $\mathrm{m} / \mathrm{z} 200$. The automatic gain control (AGC) target was set at a balanced range ( $1 \times 10^{6}$ ions). Instrument calibration was conducted daily with the use of a negative calibration solution containing sodium dodecyl sulfate, sodium taurocholate, and Ultramark ${ }^{\circledR} 1621$. Instrument settings, data acquisition, and processing were carried out by Xcalibur software (version 2.2) provided by Thermo Scientific. 


\subsubsection{TDU-MS coupling for thermal desorption of analytes}

A GERSTEL TDU connected to a cooled injection system (CIS) (GERSTEL GmbH, Mullheim, GE) was coupled to the quadrupole MS (Agilent Technologies, CA, USA) using a deactivated fused silica column (5 m length, $0.25 \mathrm{~mm}$ ID; Supelco,Bellefonte, PA, USA) as a transfer line between the injection system and the mass analyzer. Helium was used as a carrier gas, and the flow rate was setto $4 \mathrm{~mL} \mathrm{~min}^{-1}$ with a split ratio of 20:1. The column temperature was kept at $175^{\circ} \mathrm{C}$ during the analyses using a column oven Agilent 6890 GC. TF-SPME membranes (DVB-PDMS)were desorbed in the TDU with a desorption temperature program as follows: initial temperature was set $t o 40^{\circ} \mathrm{C}$ and ramped to $200^{\circ} \mathrm{C}$ at a heating rate of $700{ }^{\circ} \mathrm{C} \mathrm{min}^{-1}$, then held for $2 \mathrm{~min}$ to ensure complete desorption of analytes. During the thermal desorption process, the CIS was kept at $-50^{\circ} \mathrm{C}$, and desorbed analytes were cryo-focused in the CIS liner. Then, the CIS was heated to $275^{\circ} \mathrm{C}$ at a rate of $12{ }^{\circ} \mathrm{C} \mathrm{s}^{-1}$, and held for $2 \mathrm{~min}$ at the given final temperature. The quantification was performed using the peak area of thesignal collectedfromeach selected molecular ion.

\subsection{Method development}

\subsubsection{High throughput TF-SPME for solvent desorption and LC-MS analysis}

\subsubsection{Optimization of TF-SPME}

All method development steps for solvent desorption were performed on a Concept 96 manual unit[21],which provides parallel sample preparations of up to 96 samples with the use of a multi-well plate; consequently, in a single experiment, the best coating and desorption solvent combination could be selected. Moreover, the compact size of the manual unit allows for portability, which, together with the simplicity of the proposed protocol, makes the unit very convenient for on-site routine analysis. In order to evaluate the best coating and desorption solvent for the developed method, analytes were spiked into PBS to obtain $50 \mathrm{ng} \mathrm{mL}{ }^{-1}$ final concentrations. For this purpose, five types of coatings (HLB, PS-DVB-WAX, C18, SAX, and mixture of HLB-SAX (50/50, w/w)) and four types of desorption solutions (Solvent A: $\mathrm{ACN} / \mathrm{MeOH} / \mathrm{H}_{2} \mathrm{O}(40 / 40 / 20, \mathrm{v} / \mathrm{v} / \mathrm{v}$ ) acidified to contain $0.1 \%(\mathrm{v} / \mathrm{v}) \mathrm{FA}$; solvent $\mathrm{B}$ : $\mathrm{ACN} / \mathrm{H}_{2} \mathrm{O}(80 / 20, \mathrm{v} / \mathrm{v})$; solvent $\mathrm{C}$ : 
$\mathrm{ACN} / \mathrm{H}_{2} \mathrm{O}(80 / 20, \mathrm{v} / \mathrm{v})$ acidified with FA to contain $0.5 \%(\mathrm{v} / \mathrm{v})$; solvent D: ACN/MeOH $(80 / 20, v / v)$ were tested for selection of the best extraction phase and solvent. In these evaluations, extractions were performed from $1.5 \mathrm{~mL}$ samples in triplicate for $60 \mathrm{~min}$ at room temperature, and using $850 \mathrm{rpm}$ agitation speed. Immediately after each extraction, a $2 \mathrm{~s}$ static washing step was conducted with $1.5 \mathrm{~mL}$ water to remove any loosely retained matrix components. Next, analytes were desorbed into $1.5 \mathrm{~mL}$ of the abovementioned solvent mixtures for $60 \mathrm{~min}$, using an agitation speed of $850 \mathrm{rpm}$. A second desorption with the same conditions was applied in order to calculate the carry-over in each extraction phase-solvent pair. Using the best coating and solvent combination, further optimizations of the experimental parameters were performed. The effect of $\mathrm{pH}$, salt addition, and organic modifier, as well as extraction and desorption time profiles were among the investigated parameters.

\subsubsection{Validation of the method for seawater analysis}

For validation experiments, Mediterranean seawater collected from Messina, Italy, as well as synthetic seawater prepared in laboratory were used. Seawater samples were stored upon arrival in plastic bottles at $+4{ }^{\circ} \mathrm{C}$ until use. The same procedure was used for synthetic seawater prepared in laboratory.

The first step of validation involved verifying that matrix-to-matrix variations of seawater did not affect the performance of the method. For this purpose, seawater samples were first confirmed to be free from tested FBAs by performance of blank sample extractions with the developed high throughput TF-SPME method followed by LC-MS analysis. Next, external SPME calibrations were constructed by application of the developed method to seawater and synthetic seawater, and examination of deviations between the obtained slopes.

In order to determine figures of merit for the method, external SPME calibration solutions were prepared in synthetic seawater and Mediterranean seawater in a range of 0.1-1000 ng $\mathrm{mL}^{-1}$ of FBAs. Each calibration point was extracted in triplicate with the developed high throughput TF-SPME method, followed by LC-MS analysis. Before LCMS analysis, in order to not exceed the upper limit of the linear response of the 
instrument, the calibration samples, which had been spiked to contain more than $100 \mathrm{ng}$ $\mathrm{mL}^{-1}$ of FBA, were diluted ten times with their corresponding desorption solution.

For determination of method precision, target analytes were spiked at various concentrations $\left(0.5,5,50\right.$ and $\left.500 \mathrm{ng} \mathrm{mL}^{-1}\right)$, representing-low,-mid, and -high portions of the constructed calibrations. These extractions were performed in twelve replicates for inter-blade repeatability, and in three replicates for intra-blade repeatability, both in Mediterranean seawater and synthetic seawater.

For determination of method accuracy, the same levels of concentrations tested for precision were used in Mediterranean seawater, and relative recoveries were calculated using the TF-SPME calibration curve for synthetic seawater. Extractions were performed in twelve replicates from seawater, and the average of three replicates was used for each point of the calibration curve.

\subsubsection{Membrane TF-SPME for TDU-MS analysis}

For direct thermal desorption to the MS, extractions of FBAs were performed with a PDMS loaded with DVB ( $5 \mu \mathrm{m}$ particles) as extraction phase, which has already been approved in several studies $[28,29]$ as a thermally stable extraction phase with a wide range of analyte coverage. Extraction experiments were performed manually in $2 \mathrm{~mL}$ amber glass vials sealed with PTFE caps. For extraction, $150 \mu \mathrm{m}$ thick membranes made of PDMS and loaded with DVB were cut to $20 \mathrm{~mm} \times 7 \mathrm{~mm}$ in size and attached to a stainless steel wire $(40 \mathrm{~mm})$ that functioned as support both for the extraction and desorption steps (Figure 1S).FBAs were extracted from $1.9 \mathrm{~mL}$ of GOM acidified with $10 \mu \mathrm{L}$ of concentrated $\mathrm{HCl}$ under $1800 \mathrm{rpm}$ agitation. Following extraction, a short $(2 \mathrm{~s}$ static) washing step was applied using $1.9 \mathrm{~mL}$ of ultrapure water. Any water droplets remaining on the membranes were removed with kimwipes prior to thermal desorption. Following the extraction, the membranes were immersed into the TDU liners by holding them from the wire supports (without detaching the wire) and the liners were placed on the autosampler for injection. Extraction time profiles and linear dynamic ranges were among the selected parameters to be evaluated to show the potential of TF-SPME-MS for 
direct thermal desorption-based analysis of FBAs.

\section{Results and discussion}

\subsection{Optimization of a TF-SPME protocol for solvent desorption and LC-MS analysis}

In order to develop a high throughput TF-SPME-based analysis method for FBAs, numerous parameters were scrutinized. A number of parameters that may affect the extraction efficiency of the target compounds using SPME were evaluated: type of extractive phase (coatings/sorbent) and desorption solvent, extraction and desorption times, as well as matrix properties, including sample $\mathrm{pH}$ and salinity.

\subsubsection{Sorbent and desorption solvent selection}

In order to obtain the best possible extraction efficiency, it is crucial that an appropriate SPME extraction phase and the right desorption solvent are chosen. Thus, as a first step, a comprehensive review of the literature was undertaken in regards to SPE materials frequently used for extraction of those compounds. In light of the most commonly reported SPE sorbents for isolation of FBAs from sample matrices [5,8,9], together with our experience in developing new coatings for multi-residue analyses, and taking into consideration the physicochemical characteristics of the analytes, five different types of TF-SPME coatings, namely, HLB, C18, SAX, HLB-SAX, PS-DVB-WAX and 50/50 $(\mathrm{w} / \mathrm{w})$ mixture of SAX-HLB, were selected to be investigated. Depending on the $\mathrm{pH}$ of the sample and the extraction phase to be used, analytes can interact via multiple types of interactions with the extraction materials, such as $\pi-\pi$ interactions, hydrogen bonding, ion exchange (electrostatic), hydrophobic interactions, as well as synergetic multiple interactions of the abovementioned interactions; as such, it is worth testing all the different chemistry-possessing extraction phases so as to select the best one in terms of highest extraction efficiency and lowest carry-over. In parallel, four different compositions of desorption solvent mixtures were evaluated for their capabilities of quantitative desorption of the analytes under study. Solvent composition details can be found in section "2.3. Method development". The obtained results for the extraction 
amounts of FBAs from aforementioned coating/solvent pairs are presented in Figure 1.As shown, the best recoveries for both FBAs were obtained with HLB, SAX-HLB, and PSDVB-WAX coatings. Particularly, PS-DVB-WAX provided superior extraction for 4FBA. However, the results from a second sequential desorption applied under the same conditions (carry-over evaluation, see Supporting InformationTable 3S)showed that the PS-DVB-WAX coating provided high carry-over in a wide range of 6-18\% (results are not shown)for the tested desorption solvents. With such high carry-over values, it is possible that false positives may be reported, or that higher concentrations of samples be determined that are in reality analyte-free or contain low concentrations, especially if they are sampled subsequently to a high concentration batch. Accordingly, this sorbent was eliminated from further consideration. Among the next best two sorbents, HLB and SAX-HLB, which provided similar extraction amounts, HLB was selected as a coating for further use due to its high recoveries and the low carry-over (see Supporting Information Table 3S) obtained with the tested solvent mixtures. In addition, the mixing of HLB with SAX was observed to not dramatically enhance the extraction of FBAs compared to HLB alone. Regarding the best solvent associated with the quantitative desorption of analytes from HLB, the recoveries in Solvent D were observed to be the highest among all tested solvents. However, evaporation during the desorption step was very high, which resulted in apparent highest recoveries due to the pre-concentration of the extract. Regarding carry-over, Solvent B was shown to provide the lowest value (less than 1\%). Out of four desorption solvents and the five extraction phases tested, $\mathrm{ACN} / \mathrm{H}_{2} \mathrm{O}(80 / 20, \mathrm{v} / \mathrm{v})$ and HLB coated blades were selected as a compromise between satisfactory results regarding repeatability (RSD 3.8-6.3\%), extraction efficiency (ca 12 $\%$ in PBS), and low carry-over (less than 1\%).

\subsubsection{Effect of sample $\mathrm{pH}$ and salinity on the extraction of FBAs}

After selection of an appropriate extraction phase and solvent, the effect of sample $\mathrm{pH}$ on extraction was evaluated. For this purpose, the $\mathrm{pH}$ of ultrapure water was adjusted to acidic, neutral, and basic $\mathrm{pHs}$ by adding necessary amounts of concentrated $\mathrm{HCl}$ (for $\mathrm{pH}$ 2.0), $\mathrm{CH}_{3} \mathrm{COOH} / \mathrm{NH}_{4} \mathrm{CH}_{3} \mathrm{COO}$ (for $\mathrm{pH} 4.5$ ), $\mathrm{NH}_{4} \mathrm{HCO}_{3} / \mathrm{HCl}$ (for $\mathrm{pH} 7.0$ ), and $\mathrm{NaOH}$ (for $\mathrm{pH}$ 9.1). Since FBAs are ionizable compounds, $\mathrm{pH}$ may play an important role on 
extraction efficiency, as exemplified by our results; as can be seen on Figure 2, extracted amounts were significantly enhanced at $\mathrm{pH}$ 2.0.This was an expected result, as these compounds exist in their neutral forms at this $\mathrm{pH}$, and thus, are more available for extraction.

On the other hand, as mentioned earlier, one of the main applications for the developed method is the determination of FBAs as tracers in produced water. Considering that seawater is frequently used as an injection fluid, and that seawaters from different sources may have different ionic strengths, the extraction efficiencies of the studied FBAs needed to be evaluated under various salt concentrations. Accordingly, experiments on the effect of ionic strength (salinity) were performed under neutral $\mathrm{pH}$ by varying the salt content of the sample. The obtained results, shown in Figure 3, revealed that extraction efficiencies in the presence of salt were enhanced with respect to extractions performed in pure water. In addition, GOM, which is representative of seawater composition, and $9 \%$ and $18 \%$ $(\mathrm{w} / \mathrm{v})$ of $\mathrm{NaCl}$ showed similar values for extraction. The presence of salt involves more water molecules around ionic salts due to the formation of hydration spheres around them, thereby reducing water available to dissolve or hydrate FBAs [30].However, further increases in salt amounts to $27 \%(\mathrm{w} / \mathrm{v})$ were observed to cause a slight decrease in recoveries. Moreover, experiments performed over different time periods revealed that recoveries can show inconsistencies under neutral $\mathrm{pH}$ levels, even with adjusted ionic strengths. Therefore, for the final method, acidification of the sample (seawater or GOM) to $\mathrm{pH}$ : 2.0 was employed for all experiments.

\subsubsection{Effect of organic solvent addition on extraction efficiency}

All produced water contains some soluble oil, increasing the complexity of the sample matrix. The effect of organic content is known to play a key role in the extraction efficiency of analytes using SPME [10].More specifically, organic components dissolved in water have shown to provide difficulties during analysis of environmental water samples[31, 32].To analyze the effect of organic solvent addition on extraction efficiency, a standard mixture of benzene, toluene, ethylbenzene and xylene (BTEX)in $\mathrm{MeOH}$ was added to GOM solutions to adjust BTEX concentrations to 1,5 , and $10 \mu \mathrm{g}$ 
$\mathrm{mL}^{-1}$.Experiments with HLB coated blades resulted in the following extraction efficiencies: $98 \%, 96 \%$, and 70\%,respectively, for $4-\mathrm{FBA}$, and $88 \%, 74 \%$, and $44 \%$, respectively, for 2,3,4,5-tetraFBA. The observed decrease in extraction efficiency upon addition of BTEX could be attributed to organic content and to solvent replacement (for the $10 \mu \mathrm{g}$ mL-1BTEX solution, $1 \%$ of the water solvent was replaced by $\mathrm{MeOH}$ ). Experiments with water replaced by $\mathrm{MeOH}$ at the $1 \%, 5 \%$, and $10 \%$ levels yield extraction efficiencies of $63 \%, 63 \%$, and $50 \%$, respectively for $4-\mathrm{FBA}$, and $35 \%, 35 \%$, and $28 \%$, respectively, for 2,3,4,5-tetraFBA. Solvent composition changes have an impact on extraction efficiencies: organic solvent can reduce distribution constants for analytes, and as it was observed here, this phenomenon could be critical even when solvent displacement levels are as low as 1\% [33-34].In cases where the organic composition of the sample matrix is dynamic, matrix normalization procedures may be necessary for quantitative analysis $[33,35]$.

\subsubsection{Extraction/desorption time profiles}

Extraction time was investigated in the range of 5 to $120 \mathrm{~min}$. The time required to reach equilibrium was approximately $60 \mathrm{~min}$ for both studied compounds (Figure 2S). Nevertheless, due to the use of a 96-well plate high-throughput configuration (which allows for advantageous parallel extractions of studied samples and standards used for the matrix-matched calibration curve), good precision can be maintained even in preequilibrium conditions; accordingly, even a5 min extraction time could be applied, provided that sufficient sensitivity is obtained. As a next step, the desorption time of

analytes was evaluated in a range of 5 to $60 \mathrm{~min}$. After 5 minutes of desorption, no significant differences in desorbed amount were observed. In order to avoid artifacts associated with small variations in desorption conditions, $10 \mathrm{~min}$ was selected as desorption time. Thus, extraction and desorption times were held at 60 and $10 \mathrm{~min}$, respectively.

\subsubsection{Validation of the proposed method for high throughput TF-SPME with LC- MS based detection}

As described in the experimental section, the validation of the developed high throughput TF-SPME method was performed using prepared synthetic seawater and Mediterranean 
seawater. Constructed TF-SPME calibrations showed good correlation coefficients in both tested matrices. Moreover, the slopes of the calibrations were similar for both synthetic seawater and real seawater. The high correlation obtained in terms of the amounts of analytes extracted from these two matrices shows that synthetic seawater can be used for the construction of a matrix-matched calibration when analyte-free real samples are unavailable (see Supporting Information, Figure 3S and 4S). This not only shows the applicability of the method for different sources of seawater (which may have slightly different amounts of salts), but also shows that synthetic seawater can be used for construction of external SPME calibration for quantification purposes (matrix matched calibration).

Method LOQs were calculated for both synthetic seawater and Mediterranean seawater, and are summarized in Table 1. Individual LOQs for each analyte were chosen as the lowest level of the standard that provided less than $20 \%$ of deviation from their nominal concentration in the constructed calibration curve. A LOQ of $1.0 \mathrm{ng} \mathrm{mL} \mathrm{m}^{-1}$ was estimated for 4-FBAin both matrices, while for 2,3,4,5-tetraFBA, LOQs of 0.5 and $1.0 \mathrm{ng} \mathrm{mL} \mathrm{m}^{-1}$ were estimated for synthetic and Mediterranean seawaters, respectively.

The precision of the method was evaluated in terms of inter-blade and intra-blade repeatability. Inter-blade precision was performed with 12 blades (for each spike level) using Mediterranean seawater spiked with both FBAs at low-, mid- and high levels corresponding to $0.5,5.0,50.0$, and $500.0 \mathrm{ng} \mathrm{mL}^{-1}$ concentrations. Intra-blade repeatability was evaluated with the use of the same 3 blades in 3 separate experiments within a day, maintaining the same parameters for all experiments. In all repeatability experiments, extractions were conducted on Mediterranean seawater spiked with both FBAs at the mentioned concentration levels, and calculations were based on the amount of analytes extracted in ng. Extracted amounts were determined using standards prepared in neat solvent. The obtained results, which are summarized in Table 2, reveal good repeatability for both compounds at 5.0,50.0, and $500.0 \mathrm{ng} \mathrm{mL} \mathrm{m}^{-1}$. On the other hand, at the lowest tested level, deviations were relatively higher, but acceptable with the exception of intra-blade evaluations for 2,3,4,5-tetraFBA, which showed more than $20 \%$ RSD. 
Method accuracy was scrutinized in Mediterranean seawater at the same concentrations used for the precision evaluation. Relative recoveries from Mediterranean seawater were determined using SPME external calibration constructed in synthetic seawater. Extractions were performed in 12 replicates (12 blades). Good correlations between extracted amounts and spike levels were obtained for both tested compounds(see Table 2 for obtained accuracies and Figure $5 \mathrm{~S}$ for typical chromatograms). For 4-FBA,a relative error of more than $20 \%$ was obtained at the low-spike level $\left(0.5 \mathrm{ng} \mathrm{mL}^{-1}\right)$; however, this was an expected result, as the LOQ for that compound was calculated to be $1.0 \mathrm{ng} \mathrm{mL}^{-1}$. Considering that internal standards corrections were not used, neither for experimental bias nor instrumental drifts, the LOQs obtained in this study show that the method is adequate for the analysis of FBA tracers at the $\mathrm{ng} \mathrm{mL}^{-1}$ level in seawater. Further improvements in LOQ values can be made with the use of a triple quadrupole instrument, or the use of better pre-concentration factors; for instance, smaller desorption volumes or evaporation-reconstitution to smaller volumes can be incorporated to the proposed method.

\subsection{TF-SPME for direct thermal desorption of FBAs to MS: a step towards direct on-site analyses}

As mentioned in the introduction of this article, GC-MS determinations are capable of providing the lowest detection limits for these compounds. However, as the carboxylic acid moiety of FBAs possesses strong interactions with the GC column stationary phase, FBAs are required to be derivatized before introduction to make them GC amenable. Considering the major drawbacks of the "gold standard", and in light of the encouraging results obtained for extraction of FBAs using TF-SPME, the applicability of the TFSPME for direct thermal desorption of FBAs to MS was pursued, thus omitting chromatographic separation. This application not only provides a derivatization-free approach for thermal desorption, it also provides flexibility for on-site analysis when a portable mass spectrometer equipped with a thermal desorption unit is available. Hence, sampling and sample preparation can be performed in a single step using TF-SPME, and determinations can be completed in field. It should be kept in mind, however, that the 
results shown in this section are only preliminary evaluations showing the potential of TF-SPME for such applications.

For the purpose of this study, a connection between the GC injector port and MS analyzer was established, as shown in Supporting Information Figure 6S.Given that the formerly described PAN glued coatings are not thermally stable, for this part of the study, wellstudied PDMS loaded with DVB were used (see Supporting Information for the images, Figure 1S) as extraction phase [28,36]. Experimental evaluation included carry-over analysis on the thermally stable TF-SPME, recoveries, extraction time profiles and external SPME calibrations.

\subsubsection{Optimization of the thermal desorption}

Although high thermal desorption temperatures in the TDU can facilitate fast desorption from the membrane, in this case, significant bleeding of PDMS and production of a high background in the collected total ion chromatographic data were observed. In cases such as this, slightly lower desorption temperatures can be used to avoid significant bleeding. However, to ensure complete thermal desorption from the thin films, relatively longer desorption times should be used to compensate for the slow desorption rate at lower temperatures. Given that the CIS was kept at $-50{ }^{\circ} \mathrm{C}$ during the entire desorption step in the TDU, any slowly desorbed analytes from the thin films were re-focused on the CIS liner prior to their introduction to the MS. In this study, a 2 min desorption at $200{ }^{\circ} \mathrm{C}$ was found to be sufficient to quantitatively desorb all analytes from the thin films. As can be seen from Figure 6S (Supporting Information), the direct coupling of the injection system to the MS with the deactivated short capillary column, together with optimized conditions, made the detection of both FBAs feasible. Accordingly, it can be concluded that desorption of these compounds from DVB-PDMS is possible. The quantitative desorption of analytes was confirmed by second and third sequential desorptions and analyses (results are not shown) for carry-over.

The recoveries of FBAs with TF-SPME were estimated by spiking the same absolute amount of analyte used in the extraction step on the membrane. As can be seen (Figure 7S), under the tested conditions, extracted and spiked FBA amounts have similar peak 
areas in the chromatograph. This indicates the exhaustive nature of the extraction under the extraction phase/sample volume ratio conditions of these experiments.

\subsubsection{Extraction time profiles}

The extraction time profiles of analytes with DVB-PDMS membranes reached a plateau at 45 min under the tested conditions (Figure 8S and 9S).However, considering the fact that the extraction reached exhaustive recoveries, the observed equilibrium should be considered only as an apparent equilibrium.

\subsubsection{TF-SPME external calibration and linear range of the extraction}

Extractions of FBAs were evaluated over a wide range of concentrations using DVBPDMS membranes in order to determine the linear range and LOQ of the method. Results are shown in Figure 10S and Figure11S. Under these working conditions, the LOQs for 4-FBA and 2,3,4,5-tetraFBAwere established to be 5 and $13 \mathrm{ng} \mathrm{mL}^{-1}$, respectively. However, it is worth mentioning that in the current system, only 1 out of 20 portions of the extracted analytes were introduced to the detection system, and in splitless injection mode, lower detection limits are expected. To overcome this, an alternative way to improve sensitivity would be to increase the sample volume; however, this was not investigated at this point, since it fell outside of the main scope of the study.

In this section, the feasibility of direct thermal desorption from TF-SPME to MS without derivatization of FBAs was demonstrated. As discussed above in this section, the TFSPME can extract and thermally release analytes quantitatively and with good repeatability.

\section{Conclusions}

In this study, we demonstrated the potential of TF-SPME as a versatile tool for determination of FBAs in brine. In order to show the applicability of TF-SPME for laboratory and on-site analysis, two approaches were pursued. The main difference between these two is in their desorption procedures, namely, automated high throughput TF-SPME using solvent desorption followed by LC-MS determination, and extraction 
with thermally stable TF-SPME membranes followed by thermal desorption and coupling to a mass spectrometer (TF-SPME-MS). The former method has the advantage of simultaneously performing automated sample preparation for up to 96 samples in laboratory. Among the various tested extraction materials, HLB particles showed superior features in terms of extraction recoveries and completeness of the desorption process (low carry-over), and consequently, were selected as the extraction phase for the TFSPME blades. The proposed method requires acidification of the sample to keep analytes in their neutral form and ensure exhaustive extraction. This method, in terms of its exhaustive recoveries, can be considered as an open bed procedure, as it does not require filtration or centrifugation steps to remove sample particulates. The results obtained from the method validation experiments revealed detection limits of $1.0 \mathrm{ng} \mathrm{mL}^{-1}$, which can be further improved with the use of smaller desorption solvent volumes, larger sample volumes, or alternatively, with the use of more sensitive instrumentation, such as a triple quadrupole mass spectrometer. Organic components dissolved in water had a negative impact on extraction efficiencies. Matrix normalization studies will be needed for analyses of produced water, as this application requires consistent extraction efficiencies for samples with varying quantities of soluble oil.

The second sample preparation approach affords the advantage of avoiding gas chromatographic separations, in addition to circumventing derivatization, which is normally needed for GC-MS based determinations of FBAs. A solvent- and derivatization- free technique opens up the possibility for direct analysis of tracers at the wellhead (on-site analysis), allowing for better sample integrity, and minimization of human error during handling and shipping of samples. In addition, more frequent measurements will enable a better characterization of the connectivity between wells, which in turn will provide information to plan injection strategies and obtain maximum oil recovery.

\section{Acknowledgements}

Ezel Boyac1, Krzysztof Gorynski, and Janusz Pawliszyn thank Entanglement Technologies, Inc. for financial support. The authors would also like to acknowledge Supelco for providing the SPE particles and HPLC column used in these studies. This 
material is based upon work supported by the National Science Foundation, under Grant No. IIP-1330903.

\section{Appendix A. Supplementary data}

\section{Supplementary data associated with this article can be found in the online version,} at $\ldots . .$.

\section{References}

[1] M.Asadi, G.M.Shook,Application of Chemical Tracers in IOR, paper \# 126029presented at the 2010 North Africa Technical Conference and Exhibition, Cairo, Egypt, 14-17 February.

[2] H. Cheng, G. M. Shook, M. Taimur, D. Varadarajan, B. R. Smith, S. Muhammad, et al.,Interwell Tracer Tests to Optimize Operating Conditions for a SurfactantField Trial: Design, Evaluation and Implications, Paper \# 144899 presented at the 2011 SPE Enhanced Oil Recovery Conference, Kuala Lumpur,Malaysia, 19-21 July.

[3] C.U. Galdiga, T. Greibrokk, Trace Analysis of Fluorinated Aromatic Carboxylic Acids in Aqueous Reservoir Fluids by HPLC,J. Liq. Chrom. \& Rel. Technol., 21 (1998)855-868.

[4] G.M. Shook, G.A. Pope and K. Asakawa, Determining Reservoir Properties and Flood Performance from Tracer Test Analysis, paper \# 124614 presented at the 2009 SPE Annual Technical Conference and Exhibition, New Orleans, LA, 4-7 October.

[5] K. Muller, A. Seubert, Separation and determination of fluorobenzoic acids using ion chromatography-electrospray mass spectrometry, J. Chromatogr. A, 1270 (2012) 96-103.

[6] C. Serres-Piole, N. Moradi-Tehrania, R. Lobinski, H. Preud'homme, Direct sensitive simultaneous determination of fluorinated benzoic acids in oil reservoir waters by ultra high-performance liquid chromatography-tandem mass spectrometry, J. Chromatogr. A 1218 (2011) 5872-5877. 
[7] R.K. Juhler, A.P. Mortensen, Analysing fluorobenzoate tracers in groundwater samples using liquid chromatography-tandem mass spectrometry. A tool for leaching studies and hydrology, J. Chromatogr. A 957 (2002) 11-16.

[8]K. Muller, A. Seubert, Ultra trace determination of fluorobenzoic acids in tap and reservoir water using solid-phase extraction and gas chromatography-mass spectrometry,J. Chromatogr. A 1260(2012) 9-15.

[9] K. Muller, A. Seubert, Ultra trace determination of fluorobenzoic acids in reservoir and ground water using isotope dilution gas chromatography mass spectrometry,Isotopes Environ. Health Stud. 50(2014) 277-284.

[10] J. Pawliszyn, Theory of solid-phase microextraction,in: Handbook of Solid Phase Microextraction, 2012 13-59.

[11] E. Cudjoe, D. Vuckovic, D. Hein, J. Pawliszyn,Investigation of the Effect of the Extraction Phase Geometry on the Performance of Automated Solid-Phase Microextraction, Anal. Chem. 81 (2009) 4226-4232.

[12] D.Vuckovic, E.Cudjoe, D.Hein, J. Pawliszyn, Automation of Solid-Phase Microextraction in High-Throughput Format and Applications to DrugAnalysis, Anal. Chem. 80(2008) 6870-6880.

[13] G.A.Gomez-Rios, J. Pawliszyn, Solid phase microextraction (SPME)-transmission mode (TM) pushes down detection limits in directanalysis in real time (DART), Chem. Commun. (Camb). 50(2014) 12937-12940.

[14] G.A. Gomez-Rios, J. Pawliszyn, Development of Coated Blade Spray Ionization Mass Spectrometry for the Quantitation of Target Analytes Present in Complex Matrices, Angew. Chem. Int. Ed. 53(2014) $1-6$.

[15] A. Rodriguez-Lafuente, F.S. Mirnaghi, J. Pawliszyn, Determination of cocaine and methadone in urine samples by thin-film solid-phase microextraction and direct analysis 
in real time (DART) coupled with tandem mass spectrometry, Anal. Bioanal. Chem.405(2013)9723-9727.

[16] O.P. Togunde, K.D. Oakes, M.R. Servos, J. Pawliszyn,Optimization of solid phase microextraction for non-lethal in vivo determination of selected pharmaceuticals in fish muscle using liquid chromatography-mass spectrometry, J. Chromatogr. A 1261(2012) 99-106.

[17] V. Bessonneau, E. Boyaci, M. Maciazek-Jurczyk, J. Pawliszyn, In vivo solid phase microextraction sampling of human saliva for non-invasive and on-site monitoring, Anal.Chim. Acta. 856 (2015) 35-45.

[18] R.A. Simões, P.S. Bonato, F.S. Mirnaghi, B. Bojko, J. Pawliszyn, Bioanalytical method for in vitro metabolism study of repaglinide using 96-blade thin-film solid-phase microextraction and LC-MS/MS, Bioanalysis 7(2015) 65-77.

[19]O.P. Togunde, E. Cudjoe, K. D. Oakes, F. S. Mirnaghi, M. R. Servos, J. Pawliszyn, Determination of selected pharmaceutical residues in wastewater using an automated open bed solid phase microextraction system, J. Chromatogr. A 1262(2012) 34-42.

[20] E. Boyac1, C. Sparham, J. Pawliszyn, Thin-film microextraction coupled to LC-ESIMS/MS for determination of quaternary ammonium compounds in water samples, Anal. Bioanal. Chem.406(2014)409-420.

[21] E. Boyac1, J. Pawliszyn, Micelle Assisted Thin-Film Solid Phase Microextraction: A New Approach for Determination of Quaternary Ammonium Compoundsin Environmental Samples, Anal. Chem. 86(2014) 8916-8921.

[22] B. Bojko, D. Vuckovic,F.S. Mirnaghi, E. Cudjoe, M. Wasowicz, A. Jerath,J. Pawliszyn, Therapeutic Monitoring of Tranexamic Acid Concentration: High-Throughput Analysis With Solid-Phase Microextraction, Ther. Drug Monit.34(2012) 31-37. 
[23] V. Bessonneau, B. Bojko, A. Azad, S. Keshavjee, S. Azad, J. Pawliszyn, Determination of bronchoalveolar lavage bile acids by solid phase microextraction liquid chromatography-tandem mass spectrometry in combination with metabolite profiling: Comparison with enzymatic assay, J. Chromatogr. A 1367(2014) 33-38.

[24] K. Gorynski, B. Bojko, M. Kluger, A. Jerath, M. Wąsowicz, J. Pawliszyn, Development of SPME method for concomitant sample preparation of rocuronium bromide and tranexamic acid in plasma, J. Pharm. Biomed. Anal. 92(2014) 183-192.

[25] E. Boyac1, K. Gorynski, A. Rodriguez-Lafuente, B. Bojko, J. Pawliszyn, Introduction of solid-phase microextraction as a high-throughput sample preparation tool in laboratory analysis of prohibited substances, Anal.Chim. Acta809(2014)69-81.

[26] N. Reyes-Garcés, B. Bojko, J. Pawliszyn, High throughput quantification of prohibited substances in plasma using thin film solid phase microextraction, J. Chromatogr A. 1374(2014) 40-49.

[27] F.S. Mirnaghi, Y. Chen, L.M. Sidisky, J. Pawliszyn, Optimization of the coating procedure for a high-throughput 96-blade solid phase microextraction system coupled with LC-MS/MS for analysis of complex samples, Anal. Chem. 83(2011) 6018-6025.

[28] R. Jiang, J. Pawliszyn, Preparation of a particle-loaded membrane for trace gas sampling, Anal. Chem. 86(2014) 403-410.

[29] F.R. Kermani, A.M. Tugulea, J. Hnatiw, V.H. Niri, J. Pawliszyn, Application of automated solid-phase microextraction to determine haloacetonitriles, haloketones, and chloropicrin in Canadian drinking water, Water Qual. Res. J. Can. 48(2013)85-98.

[30] H. Farahani, M.R. Ganjali, R. Dinarvand, P. Norouzi, Study on the performance of the headspace liquid-phase microextraction, gas chromatography-mass spectrometry in 
the determination of sorbic and benzoic acids in soft drinks and environmental water samples, J. Agric. Food Chem. 57(2009) 2633-2639.

[31] E. Gracia-Lor, J.V. Sancho, F. Hernandez, Simultaneous determination of acidic, neutral and basic pharmaceuticals in urban wastewater by ultra high-pressure liquid chromatography-tandem mass spectrometry, J. Chromatogr. A 1217(2010)622-632.

[32] Z.L. Zhang, J.L. Zhou, Simultaneous determination of various pharmaceutical compounds in water by solid-phase extraction-liquid chromatography-tandem mass spectrometry, J. Chromatogr. A1154(2007) 205-213.

[33] L. Kudlejova, S. Risticevic, D. Vuckovic, J. Pawliszyn, Theory of solid phase microextraction. In: Handbook of Solid Phase Microextraction, Chemical Industry Press, Beijing, China, 2009, pp. 128-171.

[34] S. Risticevic, H. Lord, T. Górecki, C.L. Arthur, J. Pawliszyn, Protocol for solidphase microextraction method development, Nat. Protocols 5(2010) 122-139.

[35] G. Ouyang, J. Pawliszyn, Recent developments in SPME for on-site analysis and monitoring, TrAC Trend in Anal. Chem. 25(2006) 692-703.

[36] R. Jiang, E. Cudjoe,B. Bojko, T. Abaffy, J. Pawliszyn, A non-invasive method for in vivo skin volatile compounds sampling, Anal. Chim. Acta 804 (2013) 111-119. 
Table 1. Figures of merit of the method(extraction conditions: extraction volume: 1.5 $\mathrm{mL}$, desorption volume: $1.0 \mathrm{~mL}$ ).

\begin{tabular}{c|ccc|ccc}
\hline \multirow{2}{*}{ Analyte } & \multicolumn{3}{|c|}{ Synthetic seawater } & \multicolumn{3}{c}{ Mediterranean seawater } \\
\hline 4-FBA & $\mathrm{R}^{2}$ & Equation & LOQ & $\mathrm{R}^{2}$ & Equation & LOQ \\
\hline 2,3,4,5-FBA & 0.999 & $\mathrm{y}=1.138 \mathrm{x}-1.997$ & $1.0 \mathrm{ng} \mathrm{mL}^{-1}$ & 0.999 & $\mathrm{y}=1.198 \mathrm{x}-3.262$ & $1.0 \mathrm{ng} \mathrm{mL}^{-1}$ \\
\hline
\end{tabular}


Table 2. Precision and accuracy of the method (accuracy n:12, inter-blade repeatability $\mathrm{n}: 12$, intra-blade repeatability $\mathrm{n}: 3$; extraction conditions: extraction volume: $1.5 \mathrm{~mL}$, desorption volume: $1.0 \mathrm{~mL}$ ).

\begin{tabular}{c|cc|cc|cc}
\hline $\begin{array}{c}\text { Concentration } \\
\left(\mathrm{ng} \mathrm{mL} \mathrm{L}^{-1}\right)\end{array}$ & \multicolumn{2}{|c|}{$\begin{array}{c}\text { Accuracy } \\
(\mathrm{RE} \%)\end{array}$} & \multicolumn{2}{c|}{$\begin{array}{c}\text { Inter-blade repeatability } \\
\mathrm{RSD}(\%)\end{array}$} & \multicolumn{2}{c}{$\begin{array}{c}\text { Intra-blade repeatability } \\
\text { RSD (\%) }\end{array}$} \\
\hline & 4-FBA & $2,3,4,5-\mathrm{FBA}$ & 4-FBA & $2,3,4,5$-FBA & 4-FBA & $2,3,4,5$-FBA \\
\hline 0.5 & -47 & -8 & 16 & 20 & 12 & 24 \\
5.0 & 14 & 14 & 5 & 6 & 12 & 8 \\
50.0 & -8 & 7 & 4 & 2 & 5 & 3 \\
500.0 & -15 & 2 & 3 & 3 & 6 & 3 \\
\hline
\end{tabular}


Figure 1. Extracted amount of 2,3,4,5-tetraFBA (a) and 4-FBA (b) by various sorbents from PBS (Solvent A: ACN/MeOH/ $\mathrm{H}_{2} \mathrm{O}(40 / 40 / 20, \mathrm{v} / \mathrm{v} / \mathrm{v})$ acidified to contain $0.1 \%(\mathrm{v} / \mathrm{v})$ FA; solvent B: $\mathrm{ACN} / \mathrm{H}_{2} \mathrm{O}(80 / 20, \mathrm{v} / \mathrm{v})$; solvent $\mathrm{C}: \mathrm{ACN} / \mathrm{H}_{2} \mathrm{O}(80 / 20, \mathrm{v} / \mathrm{v})$ acidified with FA to contain $0.5 \%(\mathrm{v} / \mathrm{v})$; solvent $\mathrm{D}$ : $\mathrm{ACN} / \mathrm{MeOH}(80 / 20, \mathrm{v} / \mathrm{v})$ ); (extraction conditions: concentrations of analytes in PBS: $50 \mathrm{ng} \mathrm{mL}^{-1}$, extraction and desorption volumes: 1.5 $\mathrm{mL}$, extraction and desorption times: $60 \mathrm{~min}$ ), error bars represent standard deviation for 3 blades evaluated for each experimental point).

Figure 2. Amount of 2,3,4,5-tetraFBA and 4-FBA extracted at various pHs using HLB coated blades (extraction conditions: concentrations of analytes in samples: $50 \mathrm{ng} \mathrm{mL}^{-1}$, extraction and desorption volumes: $1.5 \mathrm{~mL}$, extraction and desorption times: $60 \mathrm{~min}$ ), error bars represent standard deviation for 3 blades evaluated for each experimental point).

Figure 3. Effect of salt addition on the extraction efficiency (extraction conditions: concentrations of analytes in sample: $50 \mathrm{ng} \mathrm{mL}^{-1}$, extraction and desorption volumes: 1.5 $\mathrm{mL}$, extraction and desorption times: $60 \mathrm{~min}$ ), error bars represent standard deviation for 3 blades evaluated for each experimental point). 


\section{FIGURES}
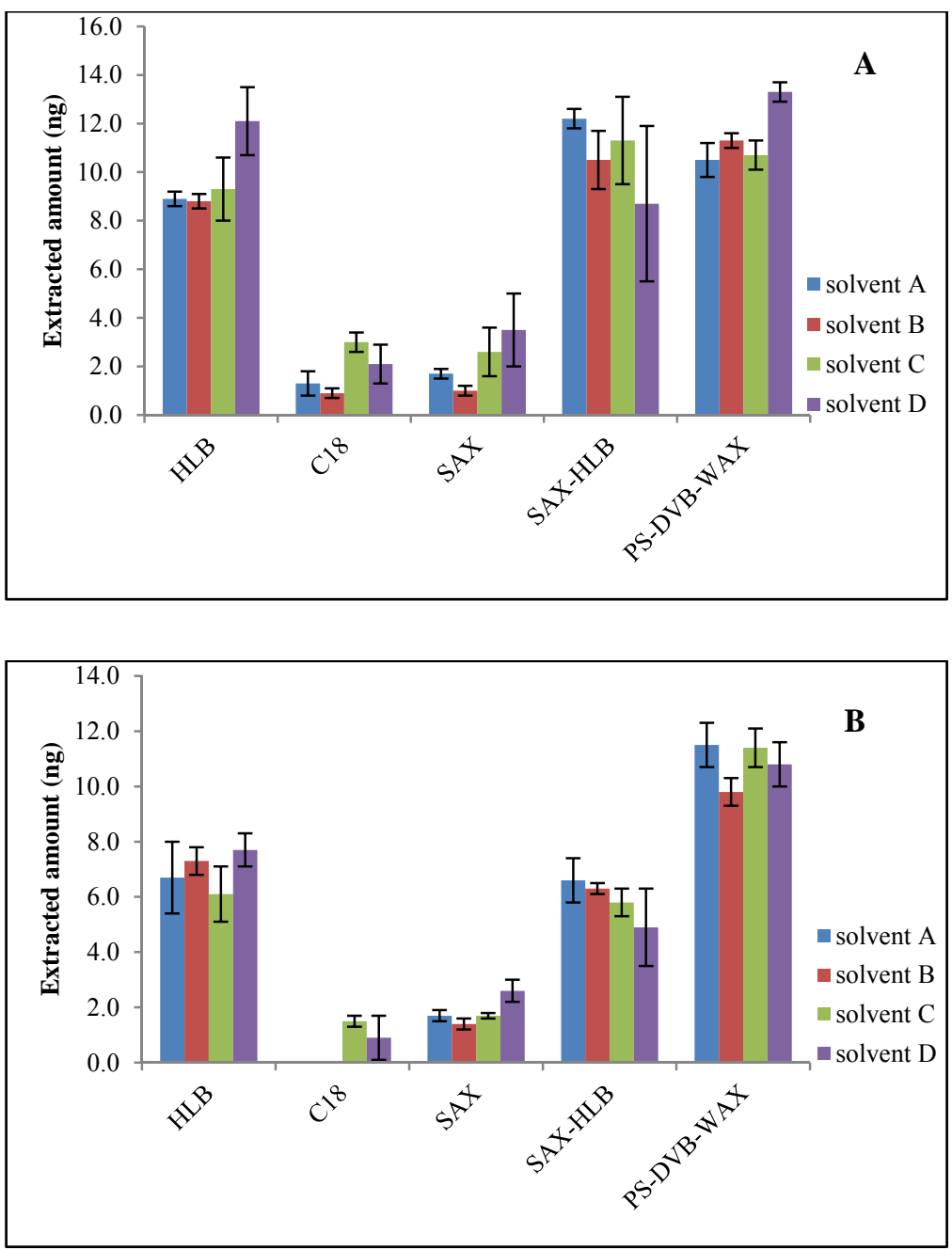

Figure 1 


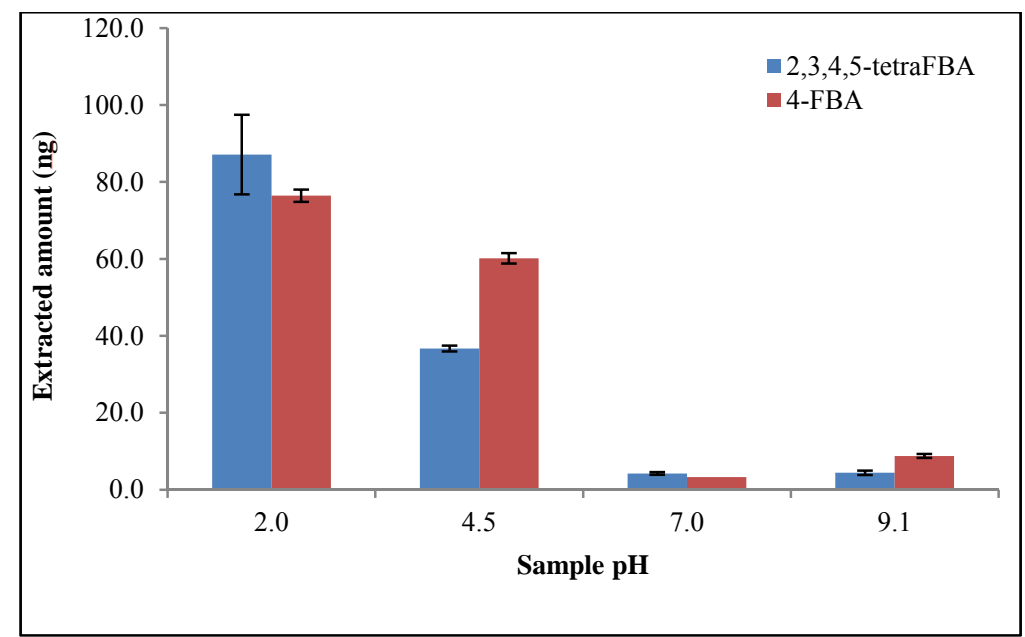

Figure 2 


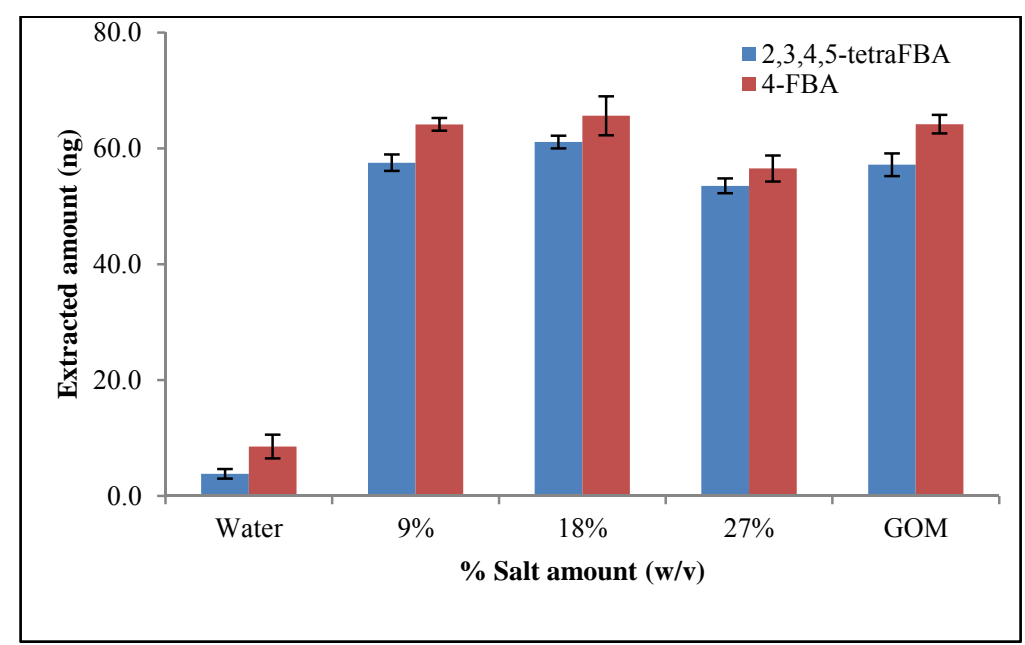

Figure 3 
Filename: $\quad$ Figures_CHROMA-S-15-02350

Directory: $\quad$ D: $\backslash$ aries

Template: $\quad$ C: $\backslash$ Users $\backslash$ abalolong.SPI-

GLOBAL $\backslash A p p D a t a \backslash$ Roaming $\backslash$ Microsoft $\backslash$ Templates $\backslash$ Normal.dotm Title:

Subject:

Author:

Ricardo Viteri

Keywords:

Comments:

Creation Date: $\quad$ 1/2/2016 12:45:00 AM

Change Number: 3

Last Saved On: $\quad$ 1/2/2016 12:48:00 AM

Last Saved By: $\quad$ Ezel Boyaci

Total Editing Time: 4 Minutes

Last Printed On: $\quad$ 8/3/2016 11:46:00 AM

As of Last Complete Printing

Number of Pages: $\quad 30$

Number of Words: $\quad$ 8,004 (approx.)

Number of Characters: 44,185 (approx.) 